\title{
Prediction of trailing-edge noise for separated turbulent boundary layers
}

\author{
Alexandre Suryadi \\ German Aerospace Center, \\ Institute of Aerodynamics and Flow Technology, \\ Lilienthalplatz 7, 38108 Braunschweig, Germany, \\ alexandre.suryadi@dlr.de
}

\begin{abstract}
This paper presents a semi-empirical low-order prediction of the trailing-edge noise of separated turbulent boundary layers. The prediction focuses on obtaining the low-frequency spectral peak of the far-field sound pressure level by modeling the measured wavenumberfrequency spectrum using regression analysis and integrating that spectrum in the manner of Howe's radiation model. Surface pressure fluctuations upstream of the trailing-edge of a DU96-W-180 blade section were measured using miniature pressure transducers, and the trailingedge noise was measured using a directional microphone. The prediction showed that the far-field sound pressure level reached its maximum below the frequency limit of the directional microphone measurement, between $400 \mathrm{~Hz}-500 \mathrm{~Hz}$ depending on the freestream velocity and the predicted spectrum varied between $\pm 0.5 \mathrm{~dB}$ given the inaccuracies of the regression model.
\end{abstract}

Keywords: prediction model, flow separation, trailing-edge noise

\section{Introduction}

With unsteady upstream conditions, such as in the atmospheric boundary layer experienced by wind turbine blades, intermittent flow separation may occur, produce more noise and increase the annoyance level of communities around a wind turbine. Brooks et al. [1] developed spectral models of airfoil self-noise for different types of noise sources among them flow separation noise. Schüle and Rossignol, [2] and [3], proposed a flow separation noise model based on the information of the boundary layer's velocity and turbulent normal stresses by using the so-called TNO surface pressure model, a popular model for the prediction of trailing-edge noise of an attached turbulent boundary layer (TBL), [4] and [5]). Measurements of the trailing-edge noise (TEN) and the surface pressure upstream of the trailing-edge were performed at a DU96-W-180 blade section in the open jet Acoustic Wind-Tunnel Braunschweig, AWB [6]. Those measurements show that the TBL separates from the geometric angle of attack $\alpha=8^{\circ}$, corresponding to a $4.6^{\circ}$ aerodynamic angle of attack. While the flow separates, the lift curve continues to increase up to the point of stall (not measured). When 
the boundary layer separates, the surface pressure autospectral density changes in two ways compared to, for example, that of the canonical zero pressure gradient TBL. (i) An increase in the lower frequency region, $f<1 \mathrm{kHz}$ and (ii) a steeper roll-off in level at mid to high frequencies [6]. The second trend is observable in the TEN measurements, using a directional microphone [3]. However, the directional microphone used in [3] is limited to $1 \mathrm{kHz} \leq f \leq 20 \mathrm{kHz}$ due to its resolution and data correction procedure, such that the spectral peak of trend (i) was not captured in the far-field sound measurement.

A recent development describes the scaling of the mean velocity profile, $U$, and turbulent shear stress, $\overline{v v}$, for separated TBLs [7]. The scaling is based on the outer layer parameters: the boundary layer thickness, $\delta$, and the local freestream velocity at $y=\delta, U_{e}$, and a non-dimensional maximum shear velocity $U_{m}=\left(\sqrt{R e_{\delta}} / U_{e}\right) \sqrt{\tau_{m} / \rho}$, where $R e_{\delta}$ is the Reynolds number based on $U_{e}$ and $\delta, \tau_{m}$ is the maximum viscous shear stress, and $\rho$ is the density. This scaling, expressed in Eqs. (1) and (2), is also suitable to scale both the surface pressure integrated power spectrum and TEN sound pressure level (SPL) spectrum.

$$
\begin{aligned}
L_{p, 1 / 3}^{\prime} & =L_{p, 1 / 3}-50 \log \left(U_{e} / a_{\infty}\right)+20 \log \left(U_{m}\right)-10 \log \left(\delta / l_{\text {ref }}\right) \\
f^{+} & =\frac{f \delta}{U_{e}} \frac{1}{U_{m}^{2}}
\end{aligned}
$$

The variables in Eqs. (1)-(2) are $L_{p, 1 / 3}: 1 / 3$-octave band sound pressure level of far-field noise, $p_{\text {ref }}=20 \mu \mathrm{Pa}, a_{\infty}$ : speed of sound, $l_{\text {ref }}$ : reference length, $1 \mathrm{~m}$. The two spectra (Figs. 7 and 8 in [7] ) show good agreement with each other in the frequency domain for the lower, more interesting frequencies for separated TBL. The far-field noise and surface pressure are related according to Howe's radiation model for classical (attached TBL) trailing-edge noise. Howe's radiation model for an observer directly above or below the noise source is

$$
S(\omega)=\frac{1}{4 \pi R^{2}}\left(\frac{\omega L}{a_{\infty}}\right) \int_{-\infty}^{\infty} \frac{\Phi_{p p}\left(k_{x}, 0, \omega\right)}{\left(k_{x}-\omega / a_{\infty}\right)} \mathrm{d} k_{x},
$$

where $\omega$ is the radial frequency, $R$ is the distance of the observer to the noise source, $L$ is the length of the wetted span, $a_{\infty}$ is the speed of sound, and $\phi_{p p}\left(k_{x}, k_{z}, \omega\right)$ is the wavenumber-frequency cross-spectrum where $k_{x}$ and $k_{z}$ are the wavenumbers in the streamwise and spanwise directions, respectively.

The goal of this study is the calculation of separated flow noise through the modeling of the cross-spectrum of the surface pressure fluctuation.

\section{Experimental setup}

All measurements were performed in the Acoustic Wind-Tunnel Braunschweig (AWB), an open jet wind tunnel with nozzle dimensions of $0.8 \mathrm{~m}$ wide and $1.2 \mathrm{~m}$ high. The maximum possible velocity is $65 \mathrm{~m} / \mathrm{s}$. The airfoil model, DU96$\mathrm{W}-180$, has a span length of $0.8 \mathrm{~m}$ and chord length of $0.3 \mathrm{~m}$, and was mounted 
(a)

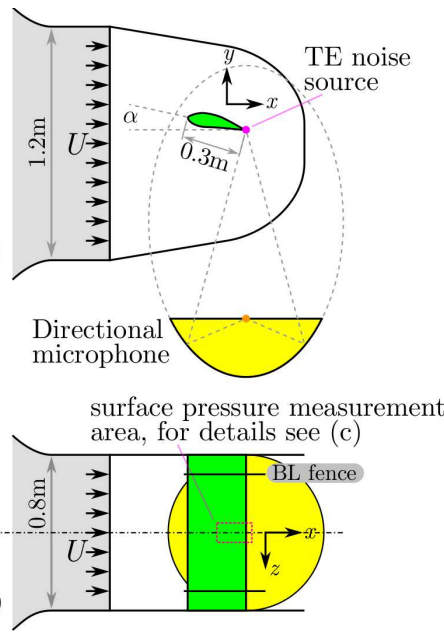

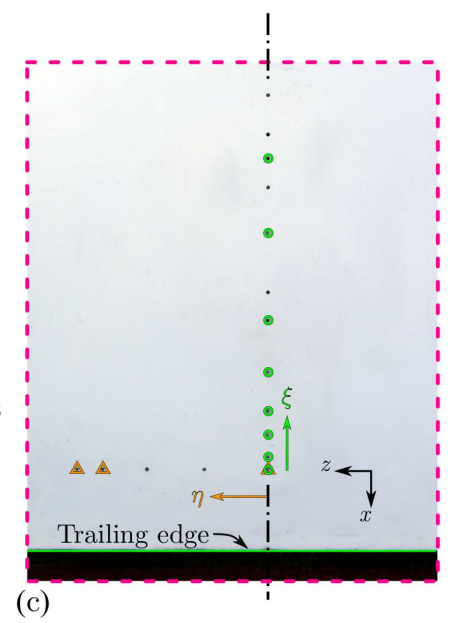

(c)

Fig. 1. Measurement setup in the AWB. (a) Side, (b) top views and (c) positions of kulite sensors used in this paper are emphasized by triangular and circular markers.

on wall extensions at either end of the nozzle width. This measurement configuration is illustrated in Fig. 1(a)-(b). Boundary layer fences $0.1 \mathrm{~m}$ away from these wall extensions are installed to limit the effect of the wall to the boundary layer development. The airfoil model was instrumented with ultra-miniature pressure sensors LQ-062-0.35barA from Kulite semiconductors. From the manufacturer's description, these sensors have a typical natural frequency of $150 \mathrm{kHz}$. A high-pass filter with a cut-off frequency of $200 \mathrm{~Hz}$ filtered the voltages from the pressure sensors. To reduce spatial attenuation these sensors were mounted under pinholes with a diameter of $0.5 \mathrm{~mm}\left(d^{+}=d u_{\tau} / \nu=29\right.$ for $\alpha=0^{\circ}$ and $U=60 \mathrm{~m} / \mathrm{s}$ ). On the suction side of the airfoil model, eight sensors were installed along the streamwise direction and, unfortunately, only three along the spanwise direction were available, Fig. 1(c). Measurements using each installation configuration were performed separately. Further description of the surface pressure sensor setup has been provided in Suryadi and Herr [6].

The far-field noise of the DU96-W-180 airfoil was measured using directional microphone [3], which provides meaningful output between $1 \mathrm{kHz}$ to $20 \mathrm{kHz}$. The turbulent boundary layers of the DU96-W-180 airfoil as mounted in the AWB separate when, $\alpha$ is larger than $8^{\circ}$. For freestream velocity $U=40 \mathrm{~m} / \mathrm{s}, 50 \mathrm{~m} / \mathrm{s}$, and $60 \mathrm{~m} / \mathrm{s}$, the pressure sensors measured the surface pressure fluctuations for $\alpha=11^{\circ}, 12^{\circ}, 13^{\circ}, 14^{\circ}$ and $14.7^{\circ}$. Measurement data of the far-field noise are available for $\alpha=11^{\circ}, 13^{\circ}$ and $14.7^{\circ}$.

\section{$3 \quad$ Result}

Figure 2 is the normalized surface pressure autospectra for the sensor positioned closest, $13 \mathrm{~mm}$ upstream, to the trailing edge. The normalization was given in [7] 


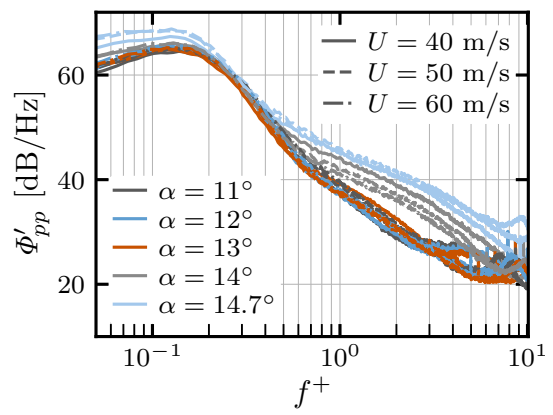

Fig. 2. (Color online) Surface pressure power spectral density for the pressure sensor $13 \mathrm{~mm}$ upstream of the trailing edge.

as $\Phi_{p p}^{\prime}\left(f^{+}\right)=U_{e} U_{m}^{2} \Phi_{p p}(f) /\left(\rho^{2} U_{e}^{4} \delta\right)$ and $f^{+}=f \delta /\left(U_{e} U_{m}^{2}\right)$ where $\Phi_{p p}$ and $\Phi_{p p}^{\prime}$ are, respectively, the dimensional and non-dimensional autospectral densities. The scaled autospectra show good agreement for $f^{+}<0.5$ for different $\alpha$ s and $U$ s with the exception of $\alpha=14.7^{\circ}$, where the scaled level is higher at $f^{+}<0.2$. This is possibly due to the angle $\alpha=14.7^{\circ}$ being very close to the stall angle that the TBL dynamics is no longer self-similar with the other, lesser $\alpha$ s.

The coherency of the surface pressure signal is given as

$$
\gamma(f, \boldsymbol{\xi})=\frac{\left|\Phi_{p p}(f, \boldsymbol{\xi})\right|}{\left[\Phi_{p p}(f ; \mathbf{x}) \Phi_{p p}(f ; \mathbf{x}+\boldsymbol{\xi})\right]^{1 / 2}},
$$

where $\Phi_{p p}(f, \boldsymbol{\xi})$ is the cross-spectral density function of $p(\mathbf{x})$ and $p(\mathbf{x}+\boldsymbol{\xi})$. The two surface pressure fluctuations have autospectra denoted as $\Phi_{p p}(f ; \mathbf{x})$ and $\Phi_{p p}(f ; \mathbf{x}+\boldsymbol{\xi})$, respectively. The vectorial notations $\mathbf{x}$ represents the spatial position of the sensor and $\boldsymbol{\xi}$ represents the distance between two sensors. The streamwise distance of two sensors with respect to the one on the mid-span and closest to the trailing-edge is denoted by $\xi$, the circular markers in Fig. 1(c). Whereas, $\eta$ is the distance of a combination of any two out of the three available sensors distributed in the spanwise and nearest to the trailing edge, triangular markers in Fig. 1(c). Statistical homogeneity of the surface pressure signal is evaluated using the autospectra in Fig. 4. The autospectra in the streamwise axis varies greatly for the two most upstream positions of the sensor because of the development of the TBL over a curved airfoil. Therefore the coherence was analyzed with respect to the last sensor in the mid-span. Whereas in Fig. 4(b) the autospectra in the spanwise axis have equal levels, which allow for the analysis in the manner previously mentioned.

Examples of the coherence spectra for streamwise and spanwise sensor separations for the measurement configuration $U=60 \mathrm{~m} / \mathrm{s}$ and $\alpha=13^{\circ}$ are shown in Figs. 3(a) and (b), respectively. The dashed line in Fig. 3 represents the exponential decay that is typical for attached TBL. For separated flow, the coherency spectra are distinctly different than for attached flow. Namely, after reaching its maximum, the coherence of separated TBL for a fixed, small value of $\xi$ is 
lost abruptly instead of following the exponential decay line. Eventually, as $\xi$ becomes larger the right-tail of the coherence spectrum follows the exponential decay. In Fig. 3(b) the phase angle $\varphi(\eta)$ was calculated using $U_{c}(f ; \xi=2 \mathrm{~mm})$. The decay rate of the spanwise coherence spectra is less than the typical value for attached TBL, relating to the presence of large spanwise structure or the so-called "mushroom"-shaped structures.

\section{Regression model of the coherency spectra}

The surface pressure power spectral densities downstream of the separation point is approximately statistically homogeneous as shown in [6] and exemplified in Fig. 4. Hence, from Eq. (4) the space-frequency coherency spectrum can be written as

$$
\Re\left\{\Phi_{p p}(f, \boldsymbol{\xi})\right\} \approx\left|\Phi_{p p}(f, \boldsymbol{\xi})\right|=\gamma(f, \boldsymbol{\xi}) \Phi_{p p}(f ; \mathbf{x})=\gamma(f, \xi) \gamma(f, \eta) \Phi_{p p}(f ; \mathbf{x}),
$$

where $\Re$ denotes the real part of the cross-spectral density. With the scaled autospectral densities shown in Fig. 2, a regression model of $\Phi_{p p}(f ; \mathbf{x})$ can be found, which for this purpose $\mathbf{x}$ is chosen to be the location of the sensor closest to trailing-edge (13 $\mathrm{mm}$ upstream of it). Thus, a regression model of $\gamma$ is needed to represent the cross-spectral density function. The expression in Eq. (5) is the same as the one given by Corcos in [8]. A regression model of the coherency spectrum in the streamwise direction $\gamma(f, \xi)$ is formulated as $\gamma(f, \xi) \approx \gamma_{\max }(\xi / \delta) \mathcal{G}\left(f^{+}, \sigma\right)$, where $\gamma_{\max }$ is the coherence decay as a function of distance and $\mathcal{G}$ is the coherence shape function.

The coherence decay for $\xi$ and $\eta$ are shown in Fig. 5. The values of $\gamma_{\max }$ were taken from coherence spectra similar to Fig. 3 after applying a moving average with a uniform kernel. The regression model of the coherence decay along the streamwise direction is

$$
\gamma_{\max }(\xi) \approx c e^{-a \xi / \delta}+(1-c) e^{-b \xi / \delta},
$$

(a)

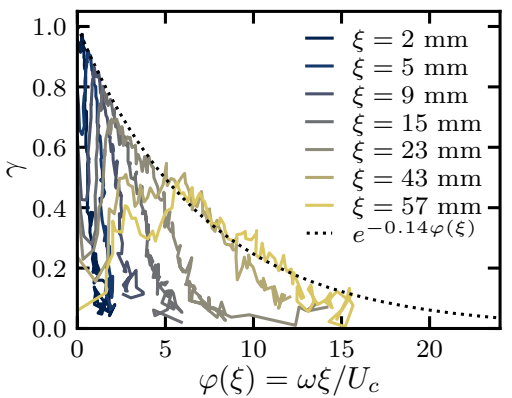

Fig. 3. (Color online) Coherence spectra of the surface pressure fluctuations (a) in the streamwise direction and (b) in the spanwise direction. Dashed line indicate exponential decay. 

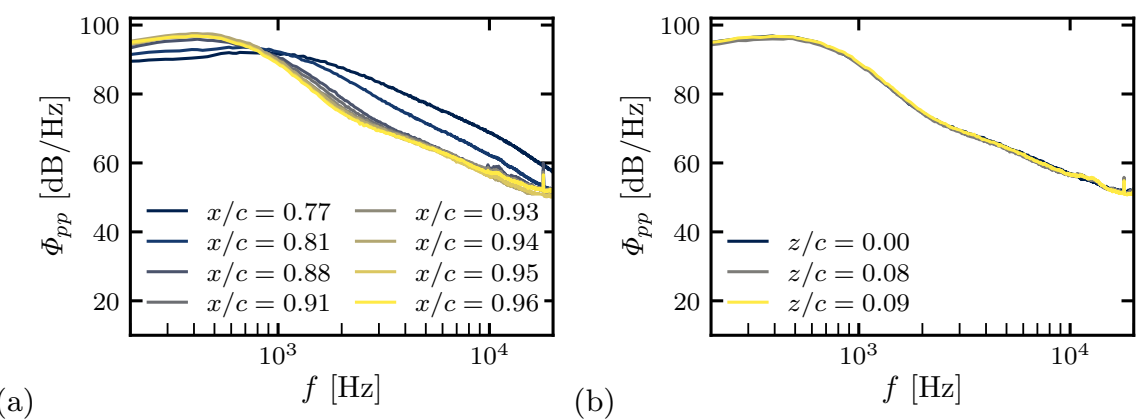

Fig. 4. (Color online)(a) Streamwise and (b) spanwise distributed autospectra at $\alpha=13^{\circ}$ and $U=60 \mathrm{~m} / \mathrm{s}$.

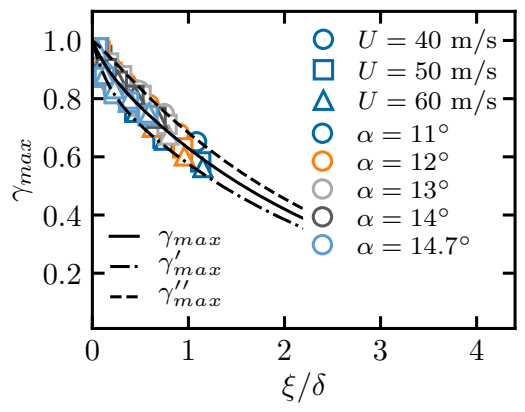

(b)

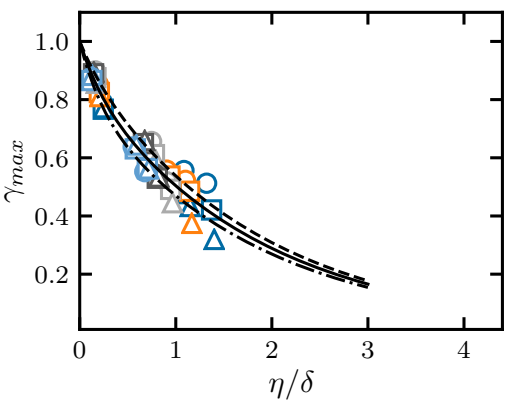

Fig. 5. (Color online) Decay of the coherence spectra as a function of distance (a) in the streamwise direction and (b) in the spanwise direction.

where $a, b$, and $c$ are constants fitted to the data. The same expression is used for the decay in the spanwise direction with $\eta$ replacing $\xi$. Using least-square approach, for the streamwise decay, $a=0.404, b=6.903$, and $c=0.942$, and for the spanwise decay $a=0.554, b=4.662$, and $c=0.875$, where $b$ contributes to the steep decay for small sensor separation distances. Equation (6) is shown in Fig. 5 as a black solid line. The black dash-dot and dashed lines indicate the spread of the data that is represented by Eq. (6) with $c^{\prime}=c-0.06$ leading to $\gamma_{\text {max }}^{\prime}$ and $c^{\prime \prime}=c+0.06$ leading to ${\gamma^{\prime \prime}}_{\text {max }}$.

The coherence shape function $\mathcal{G}\left(f^{+}, \sigma\right)$ is the coherence spectrum normalized by $\gamma_{\max }$. Streamwise and spanwise coherence shape functions are shown in Figs. 6 and 7, respectively, for selected values of $\xi$ and $\eta$. Due to smaller $\gamma_{\max }$ at large sensor separation distance, the spread of the coherence values increases. A combination of $U$ and $\alpha$ is shown, where the shape of the markers represents $U$ and the line colors represent $\alpha$. The black solid lines in Figs. 6 and 7 are the coherence shape function defined as

$$
\mathcal{G}\left(f^{+}, \sigma\right)=\frac{\gamma}{\gamma_{\max }}=\left[1-e^{-f^{+} / \tau}\right] e^{-\left(f^{+}-f_{0}^{+}\right)^{2} /\left(2 \sigma^{2}\right)}
$$


(a)
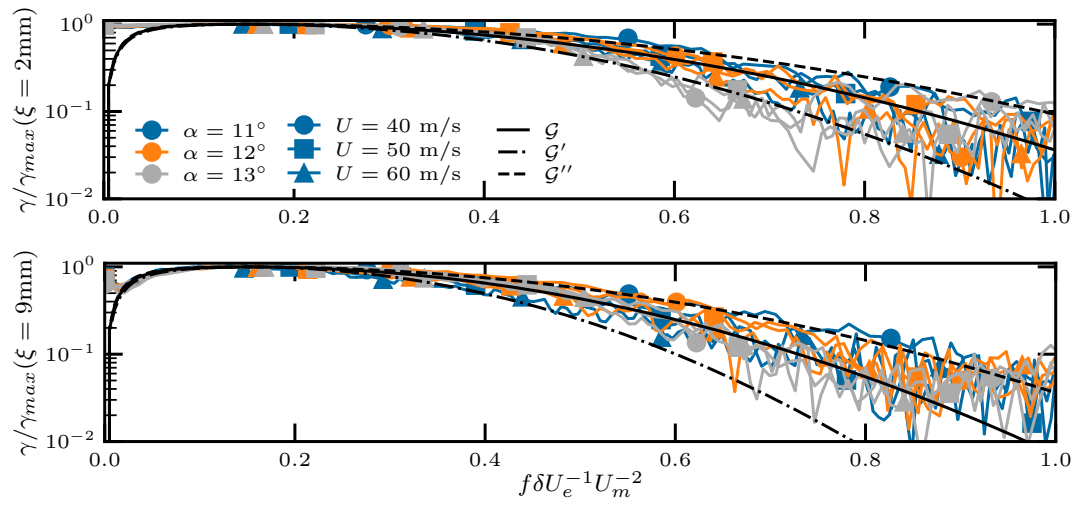

(b)

Fig. 6. (Color online) Example of coherence shape functions of two sensors $\xi$ apart. For legend explanation see text. (a) $\xi=2 \mathrm{~mm}$, and (b) $\xi=9 \mathrm{~mm}$.

where the first factor, $1-e^{-f^{+} / \tau}$, is the coherence at low-frequency with $\tau=0.02$, given arbitrarily. The reason that this factor is included is to reduce the coherence to zero at $f^{+} \propto f=0$, because $f=0$ implies coherence of two signals separated by a very large time step that they are no longer coherent or correlated. The second factor is a Gaussian curve, where $\sigma$, typically the standard deviation, denotes the size of the bell curve and $f_{0}^{+}=0.15$ is the normalized frequency of the peak of the power spectral densities (see Fig. 2). The value of $\sigma$ is expressed for each direction as $\sigma(\xi)=0.1723 \xi^{-0.1}$ and $\sigma(\eta)=0.0768 \eta^{-0.25}$ with $\xi$ and $\eta$ given in their dimensional values. However, because $\gamma / \gamma_{\max }$ is in the $f^{+}$domain, it is reasoned that $\sigma(\xi)$ and $\sigma(\eta)$ also belong to the $f^{+}$domain. The dashed-dot line in the same figures are lines drawn by Eq. (7) with $\sigma^{\prime}=\sigma-0.06$ leading to $\mathcal{G}^{\prime}$ and the dashed line is for $\sigma^{\prime \prime}=\sigma+0.06$ leading to $\mathcal{G}^{\prime \prime}$. These two lines quantify the spread of the empirical data. These and the similar lines in Fig. 5 will be used later in the study for error analysis.

Given the reason above, the wavenumber-frequency spectrum model is expressed in Eq. (8), where $\mathcal{F}_{\boldsymbol{\xi}}$ is the spatial Fourier transform, $\mathbf{k}=\left(k_{x}, k_{z}\right)$ is the wavenumber vector with elements in the streamwise and spanwise direction, respectively, and $\hat{\Phi}_{p p}\left(f^{+}\right)$is the regression function of the normalized surface pressure autospectrum.

$$
\begin{aligned}
\Phi_{p p}(f, \mathbf{k})= & \mathcal{F}_{\boldsymbol{\xi}}\left\{\Phi_{p p}(f, \boldsymbol{\xi})\right\} \\
= & \mathcal{F}_{\xi}\left\{\gamma_{\max }(\xi)\right\} \mathcal{G}_{\xi}\left(f^{+}, \sigma(\xi)\right) \mathcal{F}_{\eta}\left\{\gamma_{\max }(\eta)\right\} \mathcal{G}_{\eta}\left(f^{+}, \sigma(\eta)\right) \\
& \hat{\Phi}_{p p}\left(f^{+}\right) \frac{\left(1 / 2 \rho U_{e}^{2}\right)^{2} \delta}{U_{e} U_{m}^{2}}
\end{aligned}
$$

The Fourier transform of $\gamma_{\max }(\xi)$ is

$$
\mathcal{F}_{\xi}\left\{\gamma_{\max }(\xi)\right\}=\frac{2}{\sqrt{2 \pi}}\left[\frac{a c \delta}{a^{2}+k_{x}^{2} \delta^{2}}+\frac{b(1-c) \delta}{b^{2}+k_{x}^{2} \delta^{2}}\right]
$$



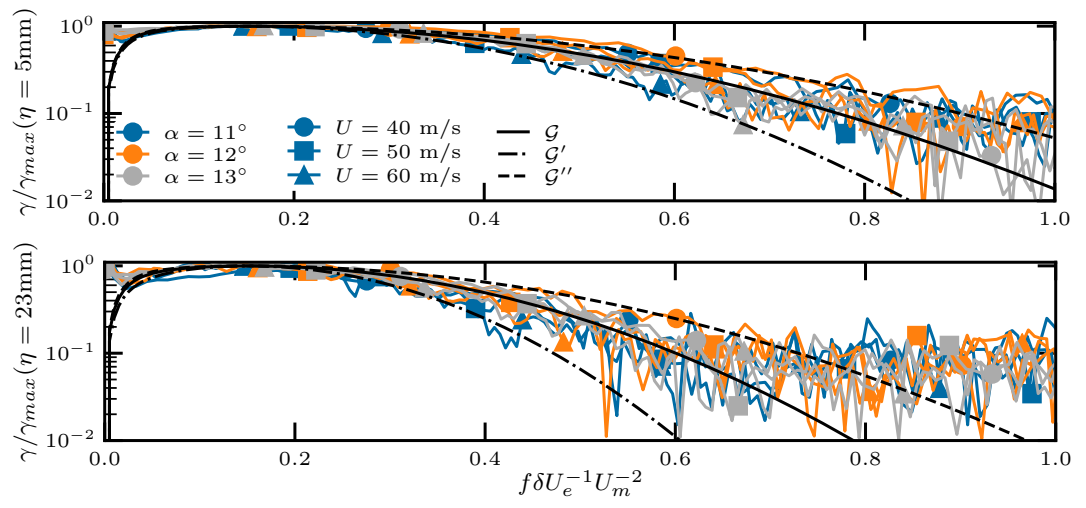

(b)

Fig. 7. (Color online) Example of coherence shape functions of two sensors $\eta$ apart. For legend explanation see text. (a) $\eta=5 \mathrm{~mm}$, and (b) $\eta=23 \mathrm{~mm}$.

and similarly for the spanwise directions by replacing $\xi$ for $\eta$ and $k_{x}$ for $k_{z}$. The values of $a, b, c$ previously mentioned apply to Eq. (9) for the respective direction of coherence decay. Because the far-field sound radiation in Eq. (3) requires the integration of the wavenumber-frequency spectra with $k_{z}=0$, the spanwise wavenumber spectrum is in the same form as Eq. (9) with the magnitude of the wavenumber equal to zero.

Low order prediction of the surface pressure autospectra and the far-field sound, as denoted by the lines, is compared with the measurements, as denoted by the markers in Fig. 8. Line and marker colors denote variations of $U$ and line and marker styles denote variations of $\alpha$. Each of the predicted autospectrum in Fig. 8(a) is a product of the integration of $\Phi_{p p}\left(k_{x}, \eta=0, f\right)$ with respect to $k_{x}$, where the integrand is equal to $\mathcal{F}\left\{\gamma_{\max }(\xi)\right\} \mathcal{G}_{\xi} \hat{\Phi}_{p p}=\int_{-\infty}^{\infty} \Phi_{p p}\left(k_{x}, k_{z}, f\right) \mathrm{d} k_{z}$. The integration of the autospectrum was done this way to avoid an oversized calculation matrix. In Fig. 8(b) the predictions produce curves in the low-frequency range where the sound pressure maxima are located. In the same manner, the surface pressure autospectra have their strongest energy content in the lowfrequency region, see Fig. 8(a). The reason the spectral peaks are predominantly at low frequency was explained by Simpson et al. [9], who measured the velocity within a separated TBL. They found that downstream of flow separation, turbulent production terms near the wall are smaller compared to attached TBL and turbulence production in the outer layer supplies the turbulent energy near the wall by turbulent diffusion.

Comparison of the prediction of the trailing-edge noise in Fig. 8(b) with that given in [3] reveal that the spectral peaks derived by the present model are between $5 \mathrm{~dB}$ to $10 \mathrm{~dB}$ higher and the frequency of the spectral peaks are $200 \mathrm{~Hz}$ - $300 \mathrm{~Hz}$ larger depending on the value of $\alpha$. Furthermore, the calculation of the TEN presented in Fig. 8(b) does not utilize the TNO surface pressure spectrum model that was derived for attached TBL. 
(a)

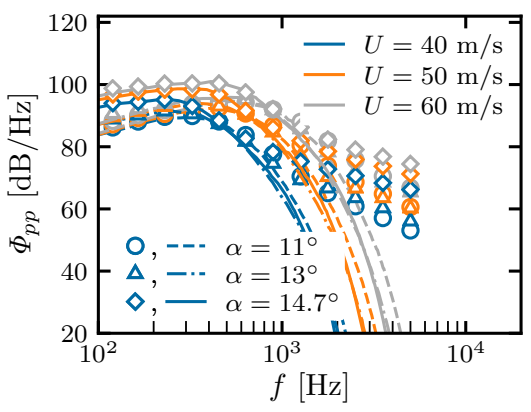

(b)

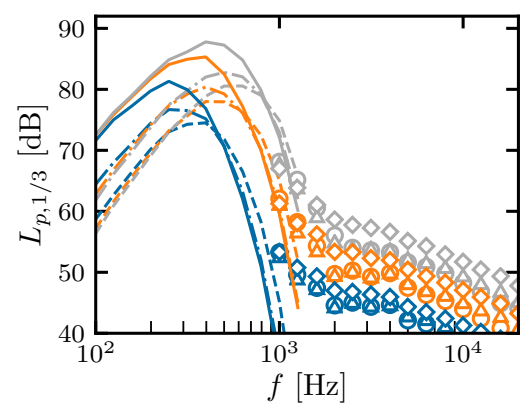

Fig. 8. (Color online) Spectral predictions based on the regression model, Eq. (8). (a) reconstructed surface pressure autospectra (smaller number of markers are shown for clarity) and (b) far-field sound pressure level spectra. For legend explanation see text.

(a)

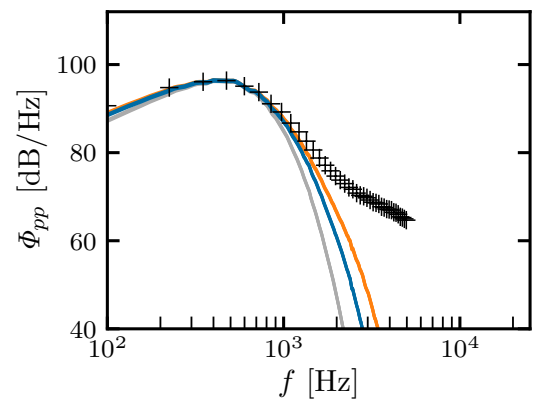

(b)

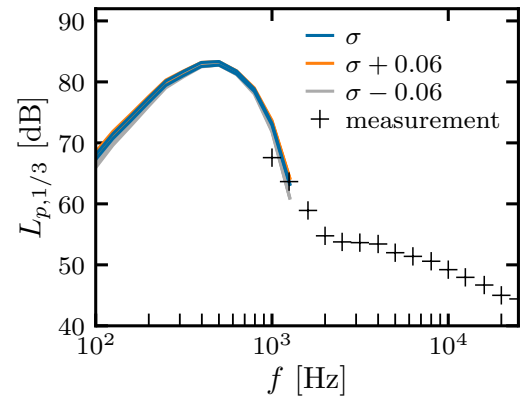

Fig. 9. (Color online) Error analysis for configuration $U=60 \mathrm{~m} / \mathrm{s}$ and $\alpha=13^{\circ}$. (a) surface pressure autospectra (smaller number of markers are shown for clarity) and (b) far-field sound pressure level spectra.

\section{Error analysis}

The robustness of the regression model is examined by substituting the values $c$ in Eq. (6) and $\sigma$ in Eq. (7) so that $\gamma_{\max }$ and $\mathcal{G}$ encompasses the spread of the empirical data. For $\gamma_{\max }$, an addition of -0.06 to $c$ leads to the curve $\gamma_{\max }^{\prime}$ and +0.06 leads to $\gamma_{\max }^{\prime \prime}$ in Fig. 5. The spread of the empirical coherence shape function is bounded by the dashed lines in Figs. 6 and 7 , where $\mathcal{G}^{\prime}=\mathcal{G}\left(f^{+}, \sigma-\right.$ $0.06)$ and $\mathcal{G}^{\prime \prime}=\mathcal{G}\left(f^{+}, \sigma+0.06\right)$ for the respective $\sigma$ of either direction. The inaccuracies of the regression model are shown in Fig. 9 for the measurement configuration $U=60 \mathrm{~m} / \mathrm{s}$ and $\alpha=13^{\circ}$. It shows that the spectral prediction is weakly dependent with $c$ and more strongly with $\sigma$. In fact lines due to the variation of $c$ are plotted in Fig. 9, but they overlap each other. Despite the variation in the coherence shape function, the spread of the sound pressure level is within $\pm 0.5 \mathrm{~dB}$. 


\section{Conclusion}

This paper presents a model to calculate the far-field sound of turbulent flow separation. The model was derived from empirical data of surface pressure fluctuations near the trailing edge. It was shown that the model reconstructed the surface pressure autospectrum in the low-frequency region, which resulted in a low-frequency spectrum for the far-field noise that complements the measurement result, which is limited to frequencies above $1 \mathrm{kHz}$. Variations in the regression model result in variations in the far-field sound of $\pm 0.5 \mathrm{~dB}$.

GE Wind Energy GmbH sponsored the present study between 2012 and 2015.

\section{References}

1. Brooks, T.F., Pope, S., Marcolini, M.A.: Airfoil self-noise and prediction. Technical Report, NASA-RP-1218 (1989).

2. Schüle, C.Y., Rossignol, K.S.:A separated flow model for semi-empirical prediction of trailing edge noise. In: Dillman, A., et al. (eds.) New Results in Numerical and Experimental Fluid Mechanics IX, vol 124, pp. 639-647, Springer, Heidelberg (2014). doi:10.1007/978-3-319-03158-3

3. Schüle, C.Y., Rossignol, K.S.: Trailing-edge noise modeling and validation for separated flow conditions. In: 19th AIAA/CEAS Aeroacoustics Conference, AIAA 2013-2008 (2013). doi:10.2514/6.2013-2008

4. Herr, M., Kamruzzaman, M.: Benchmarking of trailing edge noise computations outcome of the BANC-II workshop. In: 19th AIAA/CEAS Aeroacoustics Conference, AIAA 2013-2123 (2013). doi:10.2514/6.2013-2123

5. Herr, M., Bahr, C.J., Kamruzzaman, M.: Broadband trailing-edge noise predictions - overview of BANC-III results. In: 21st AIAA/CEAS Aeroacoustics Conference, AIAA 2015-2847 (2014). doi:10.2514/6.2015-2847

6. Suryadi, A., Herr, M.: Wall pressure spectra on a DU96-W-180 profile from low to pre-stall angles of attack. In: 21st AIAA/CEAS Aeroacoustics Conference, AIAA 2015-2688 (2015). doi:10.2514/6.2015-2688

7. Suryadi, A.: Kinematic and acoustic similarities of separated turbulent boundary layers. In: 24th AIAA/CEAS Aeroacoustics Conference, AIAA 2018-3937 (2018). doi:10.2514/6.2018-3937

8. Corcos, G.M.: The structure of the turbulent pressure field in boundary-layer flows. J. Fluid Mech. 18(3), pp. 353-378 (1964).doi:10.1017/S002211206400026X

9. Simpson, R.L., Chew, Y.T., Shivaprasad, B.G.: The structure of a separating turbulent boundary layer. Part 2. Higher-order turbulence results. J. Fluid Mech. 113, pp. 53-73 (1981).doi:10.1017/S0022112081003406 